\title{
Splashing transients of 2D plasmons launched by swift electrons
}

\author{
Xiao Lin, ${ }^{1,2}$ Ido Kaminer, ${ }^{2 *}$ Xihang Shi, ${ }^{3}$ Fei Gao, ${ }^{3}$ Zhaoju Yang, ${ }^{3}$ Zhen Gao, ${ }^{3}$ Hrvoje Buljan, ${ }^{4}$ \\ John D. Joannopoulos, ${ }^{2}$ Marin Soljačić, ${ }^{2}$ Hongsheng Chen, ${ }^{1 *}$ Baile Zhang ${ }^{3,5 *}$
}

Launching of plasmons by swift electrons has long been used in electron energy-loss spectroscopy (EELS) to investigate the plasmonic properties of ultrathin, or two-dimensional (2D), electron systems. However, the question of how a swift electron generates plasmons in space and time has never been answered. We address this issue by calculating and demonstrating the spatial-temporal dynamics of 2D plasmon generation in graphene. We predict a jet-like rise of excessive charge concentration that delays the generation of 2D plasmons in EELS, exhibiting an analog to the hydrodynamic Rayleigh jet in a splashing phenomenon before the launching of ripples. The photon radiation, analogous to the splashing sound, accompanies the plasmon emission and can be understood as being shaken off by the Rayleigh jet-like charge concentration. Considering this newly revealed process, we argue that previous estimates on the yields of graphene plasmons in EELS need to be reevaluated.

\section{INTRODUCTION}

Since the first successful confirmation of surface plasmons $(1,2)$ on the platform of thin metal films, swift electrons have long been used to investigate plasmonic properties of ultrathin, or two-dimensional (2D), electron systems, including graphene plasmons recently (3-10). Electron energy-loss spectroscopy (EELS) $(1,3,11)$, which uses swift electrons as a probe, has been an indispensable tool in studying $2 \mathrm{D}$ plasmons. On the other hand, despite the long history of studies of $2 \mathrm{D}$ plasmons $(1,2)$, the dynamic mechanism of how a swift electron launches $2 \mathrm{D}$ plasmons has never been clearly revealed.

This is because the impact of an electron will generate not only plasmons but also photons [the so called "transition radiation" $(12,13)$ ], whose emission cannot be achieved at a single space-time point but demands a finite space-time region. Historically, the concepts of "formation time" and "formation zone" [that is, "it takes a relatively long time and, therefore, a long distance for an energetic electron to create a photon," as retailed by Uggerhøj (14)] were first presented by TerMikaelian in Landau's seminar in 1952 (15). Landau strongly opposed these concepts at first (15), but soon realized their correctness and significance and further developed them in the LandauPomeranchuk-Migdal effect, which was experimentally confirmed 40 years later. The existence of formation time and formation zone reflects our ignorance about the exact moment and location at which a photon is generated. In the early days of the quantum uncertainty principle, Bohr already commented on the impossibility of describing the electronphoton interaction "without considering a finite space-time region" (16). Later, it was Ginzburg and colleagues $(12,13)$ who included the formation time and formation zone with mathematical form into the classical framework of transition radiation (established by Ginzburg and Frank in 1945) while still admitting that "comparatively little is known."

\footnotetext{
${ }^{1}$ State Key Laboratory of Modern Optical Instrumentation, Zhejiang University, Hangzhou 310027, China. ${ }^{2}$ Department of Physics, Massachusetts Institute of Technology, Cambridge, MA 02139, USA. ${ }^{3}$ Division of Physics and Applied Physics, School of Physical and Mathematical Sciences, Nanyang Technological University, Singapore 637371, Singapore. ${ }^{4}$ Department of Physics, University of Zagreb, Bijenička c. 32, 10000 Zagreb, Croatia. ${ }^{5}$ Centre for Disruptive Photonic Technologies, Nanyang Technological University, Singapore 637371, Singapore.

*Corresponding author. Email: kaminer@mit.edu (I.K.); hansomchen@zju.edu.cn (H.C.); blzhang@ntu.edu.sg (B.Z.)
}

Although the above space-time discussions in the context of photon emission have lasted for decades, similar discussions have never been conducted on the generation of plasmons in EELS experiments. This explains why the spatiotemporal process of $2 \mathrm{D}$ plasmon launching by a swift electron remains elusive after a long research history.

In this paper, we introduce the concepts of formation time and formation zone into plasmon generation in EELS. On the platform of a graphene monolayer, we show within the framework of classical electrodynamics the dynamical process of $2 \mathrm{D}$ plasmon launching by a swift electron affecting on the graphene monolayer (see movie S1 for this process). We link this dynamical process during the formation time of graphene plasmons to the deep-water hydrodynamic splashing phenomenon, in which a picosecond jet-like rise of excessive charge concentration is formed on graphene as an analog of the "Rayleigh jet" (also called "Worthington jet") in hydrodynamic splashing $(17,18)$. In this newly revealed physical process, a significant part of electromagnetic energy has already been dissipated before graphene plasmons are generated. In view of this consideration, we show that previous estimates of graphene plasmon yields (3-6) from the electron energy-loss (EEL) spectra need to be reevaluated. Note that although we adopt graphene as the platform, our analysis is general in any $2 \mathrm{D}$ electron system. In addition, although the formal similarity of dispersion between hydrodynamic water waves and $2 \mathrm{D}$ electron systems (19-23) [including graphene plasmons $(24,25)$ ] has been investigated with some hydrodynamic wave-like phenomena predicted (26-29), the analog to the splashing phenomenon has never been discussed.

\section{RESULTS}

\section{Modeling of 2D plasmon launching by swift electrons}

The model of calculation is schematically shown in Fig. 1. We consider a swift electron with charge $q$, moving with a velocity $\bar{v}=\hat{z} v=\hat{z} \beta c$, where $c$ is the speed of light in free space. Because the energy loss of the electron (coupled to plasmons and transition radiation) is much lower than the electron's kinetic energy, the electron's velocity is treated as constant (13). The space-time dependence of the current density due to the electron is classically described as $\bar{J}^{q}(\bar{r}, t)=$ $\hat{z} J_{z}^{q}(\bar{r}, t)=\hat{z} q v \delta(\bar{r}-\bar{v} t)$, in which $t=0$ when the electron goes through the origin. A graphene layer is located at the interface between medium $1(z<0)$ with permittivity $\varepsilon_{1 r} \varepsilon_{0}$ and medium $2(z>0)$ with 


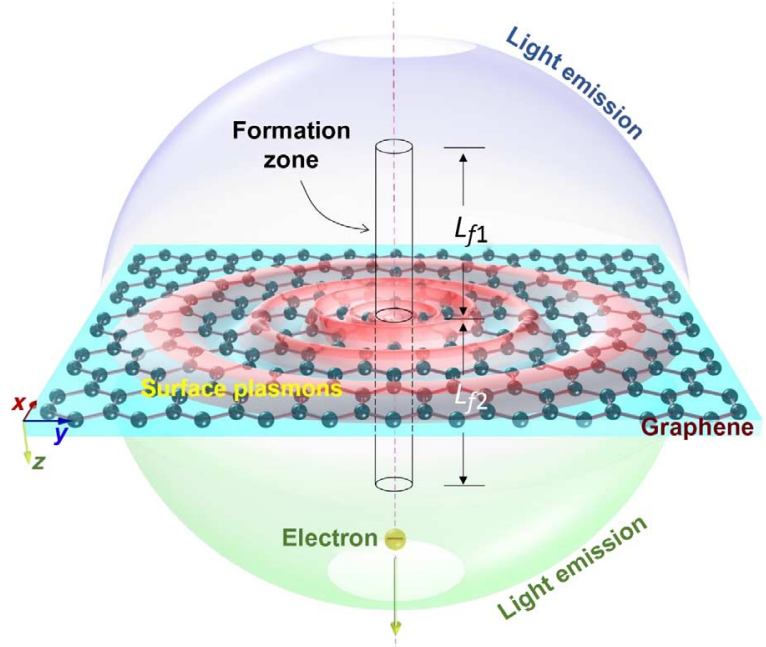

Fig. 1. Schematic of 2D plasmons launching with a swift electron penetrating through a graphene monolayer. $L_{f 1}$ and $L_{f 2}$ are the lengths of the formation zone in the region above and below the graphene layer, respectively.

permittivity $\varepsilon_{2 r} \varepsilon_{0}$, where $\varepsilon_{0}$ is the permittivity of free space. To simplify the analysis, we set mediums 1 and 2 to be free space. As a typical 2D electron system, graphene can be characterized by two macroscopic variables (24): the deviation of the electron density from its average value $\delta n(\bar{r}, t)$ and the associated current density $\delta \bar{J}(\bar{r}, t)$, which depends on graphene's surface conductivity $\sigma_{s}$. Here, we calculate graphene's surface conductivity based on the Kubo formula (see section S2) $(30,31)$ and set the relaxation time to $\tau=0.5 \mathrm{ps}$ and the chemical potential in graphene to $\mu_{c}=0.4 \mathrm{eV}$ [well within experimental capabilities $(32,33)$ ]. Because of the rotational symmetry of the system, all fields must be transverse magnetic and thus can be characterized by an electric field component $E_{z}$ in the $\hat{z}$ direction. By decomposing all the quantities into Fourier components in time and in the coordinates $\bar{r}_{\perp}=\hat{x} x+\hat{y} y$ with corresponding wavevectors $\hat{\kappa}_{\perp}=\hat{x} \kappa_{x}+\hat{y} \kappa_{y}$, we have

$$
\begin{aligned}
& J_{z}^{q}(\bar{r}, t)=\int j_{\bar{\kappa}_{\perp}, \omega}^{q}(z) e^{i\left(\bar{\kappa}_{\perp} \cdot \bar{r}_{\perp}-\omega t\right)} d \bar{\kappa}_{\perp} d \omega \\
& E_{z}(\bar{r}, t)=\int E_{\bar{\kappa}_{\perp}, \omega}(z) e^{i\left(\bar{\kappa}_{\perp} \cdot \bar{r}_{\perp}-\omega t\right)} d \bar{\kappa}_{\perp} d \omega
\end{aligned}
$$

By writing the fields due to the electron field as $E_{\overline{\mathrm{K}}_{1}, \omega}^{q}$ and the radiation fields in regions of $z<0$ and $z>0$ as $E_{\overline{\mathrm{K}}_{\perp}, \omega}^{1}$ and $E_{\overline{\mathrm{K}}_{\perp}, \omega^{\prime}}^{2}$ we can get the total fields in regions of $z<0$ and $z>0$ as $E_{\overline{\mathrm{K}}_{\perp}, \omega}^{q}+E_{\overline{\mathrm{K}}_{\perp}, \omega}^{1}$ and $E_{\overline{\mathrm{K}}_{\perp}, \omega}^{q}+E_{\overline{\mathrm{K}}_{\perp}, \omega}^{2}$, respectively. Derivation from matching boundary conditions at the graphene plane shows phase relations of $E_{\bar{\kappa}_{1}, \omega}^{q} \sim \exp (i \omega z / v)$ and

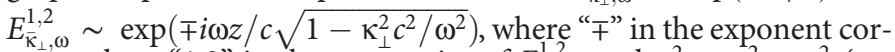
responds to "1,2" in the superscript of $E_{\bar{\kappa}_{\perp}, \omega}^{1,2}$ and $\kappa_{\perp}^{2}=\kappa_{x}^{2}+\kappa_{y}^{2}$ (see section S2).

\section{Formation zone and formation time}

Ginzburg estimated the length of the formation zone (denoted as "formation length" $L_{f}$ ) for photon emission in transition radiation based on the following considerations (13). Inside the formation zone, the total energy of the fields $E_{\overline{\mathrm{K}}_{\perp}, \omega}^{q}+E_{\overline{\mathrm{K}}_{\perp}, \omega}^{1,2}$ is proportional to $\left(E_{\bar{\kappa}_{\perp}, \omega}^{q}+E_{\bar{\kappa}_{\perp}, \omega}^{1,2}\right)^{2}$. The length $L_{f}$ describes a boundary at which the total energy becomes practically equal to the sum of the energy of the electron field [that is, $\left(E_{\overline{\mathrm{K}}_{\perp}, \omega}^{q}\right)^{2}$ ] and the energy of the radiation field [that is, $\left.\left(E_{\overline{\mathrm{K}}_{\perp}, \omega}^{1,2}\right)^{2}\right]$ meaning that the electron field and the radiation field are separated from each other. That is, the interference term $E_{\overline{\mathrm{K}}_{\perp}, \omega}^{q} \cdot E_{\overline{\mathrm{K}}_{\perp}, \omega}^{1,2}$ must play a trivial role. Ginzburg thus set the phase difference of $2 \pi$ between $E_{\overline{\mathrm{K}}_{\perp}, \omega}^{q}$ and $E_{\overline{\mathrm{K}}_{\perp}, \omega}^{1,2}$ to determine the length $L_{f}$. That is, $\frac{\omega L_{f}}{v} \pm \frac{\omega L_{f}}{c} \sqrt{1-\frac{\kappa_{\perp}^{2} c^{2}}{\omega^{2}}}=2 \pi$, which gives

$$
L_{f 1}=\frac{2 \pi}{\left|\frac{\omega}{v}+\frac{\omega}{c} \sqrt{1-\frac{\kappa_{1}^{2} c^{2}}{\omega^{2}}}\right|}, L_{f 2}=\frac{2 \pi}{\left|\frac{\omega}{v}-\frac{\omega}{c} \sqrt{1-\frac{\kappa_{1}^{2} c^{2}}{\omega^{2}}}\right|}
$$

where $L_{f 1}$ and $L_{f 2}$ correspond to the length of the formation zones in regions of $z<0$ and $z>0$, respectively (see Fig. 1). Apparently, the formation length depends not only on the frequency of emitted photons and the swift electron's velocity but also on the emission angle of photons. As a numerical example, with the electron's velocity $v=0.8 c$, to emit a photon at frequency $10 \mathrm{THz}$ in the backward $-\hat{z}$ direction $\left(\kappa_{\perp}^{2}=0\right)$, it takes the electron about $\frac{L_{f 1}}{v}=0.06$ ps to accomplish it. For photon emission in the forward $+\hat{z}$ direction $\left(\kappa_{\perp}^{2}=0\right)$ at the same frequency, it takes about $\frac{L_{f 2}}{v}=0.5 \mathrm{ps}$. These time intervals of 0.06 and 0.5 ps are considered as formation times for the backward and forward photon emission at $10 \mathrm{THz}$.

Ginzburg's estimation only applies to photon emission with the condition $\kappa_{\perp}^{2}<\omega^{2} / c^{2}$ to satisfy the square root in Eq. 3. Yet, it is well known that surface plasmons have $\kappa_{\perp}^{2}>\omega^{2} / c^{2}$. Compared to the electron-photon interaction as analyzed by Ginzburg, in the interaction between the electron and surface plasmons, only the electron field has phase variation along the $z$ axis, whereas the surface plasmons have zero phase variation. Therefore, along the line of Ginzburg's thought, the phase difference of $2 \pi$ between $E_{\bar{\kappa}_{\perp}, \omega}^{q}$ and $E_{\overline{\mathrm{K}}_{\perp}, \omega}^{1,2}$ should only come from $E_{\overline{\mathrm{K}}_{\perp}, \omega}^{q}$, that is, $\frac{\omega L_{f}}{v}=2 \pi$. This gives

$$
L_{f 1}=L_{f 2}=\frac{2 \pi v}{\omega}
$$

Numerically, it means that it will take the electron about $\left(L_{f 1}+L_{f 2}\right) /$ $v=0.2$ ps to launch a $2 \mathrm{D}$ plasmon at $10 \mathrm{THz}$. The value $0.2 \mathrm{ps}$ is considered as the formation time for the $2 \mathrm{D}$ plasmon at $10 \mathrm{THz}$.

\section{Transients of photon emission}

It is interesting to see what happens during the formation time of $2 \mathrm{D}$ plasmons when the electron goes through the formation zone, as schematically illustrated in Fig. 1. The dynamical process of photon emission and 2D plasmon launching by a swift electron moving with $v=0.8 c$ (or kinetic energy $340 \mathrm{keV}$ ), which can be conveniently realized in modern electron microscope systems $(1,34)$, is shown in movie S1. (The relatively large velocity considered in this simulation shows that our theoretical analysis is not limited to low electron speeds.) The bandwidth of calculation is from 0 to $20 \mathrm{THz}$, as justified in fig. S3. Figure 2 shows the temporal evolution of photon emission. When the incident electron moves close to the graphene interface (Fig. 2A), its evanescent field touches graphene, expelling the conduction electrons from the vicinity of the swift electron's trajectory. Because of electromagnetic induction, the induced surface current in turn blocks the penetration of the evanescent electron field by accumulating fields on the upper side of graphene (Fig. 2, A and B). Immediately after the electron crosses the 
graphene (Fig. 2, B and C), the fields previously accumulated on the upper side have lost direct contact with the electron and thus are "shaken off" into the upper space. Meanwhile, the insufficient fields on the lower side of graphene need to recover their strength in the presence of the electron and thus "shake off" radiation into the lower space (Fig. 2, C and D). It can be seen that, at the central frequency $(10 \mathrm{THz})$ of the calculation bandwidth, the formation time of $0.06 \mathrm{ps}$ for the backward radiation is consistent with Fig. 2 ( $\mathrm{A}$ and $\mathrm{B}$ ) and that of 0.5 ps for the forward radiation is consistent with Fig. 2 (C and D).

\section{Transients of 2D plasmon launching}

Of greater interest is the charge motion on graphene itself. We plot the dynamics of the deviation of electron density from its average value $\delta n(\bar{r}, t)$ on graphene during the penetration of the swift electron in Fig. 3. Similarities to the hydrodynamic splashing scenario caused by a little droplet falling on a 2D liquid surface are evident, as described below.

First, in hydrodynamics $(17,18)$, when a small droplet affects a calm, deep-water surface, a crater is formed first and water splashes to the side.

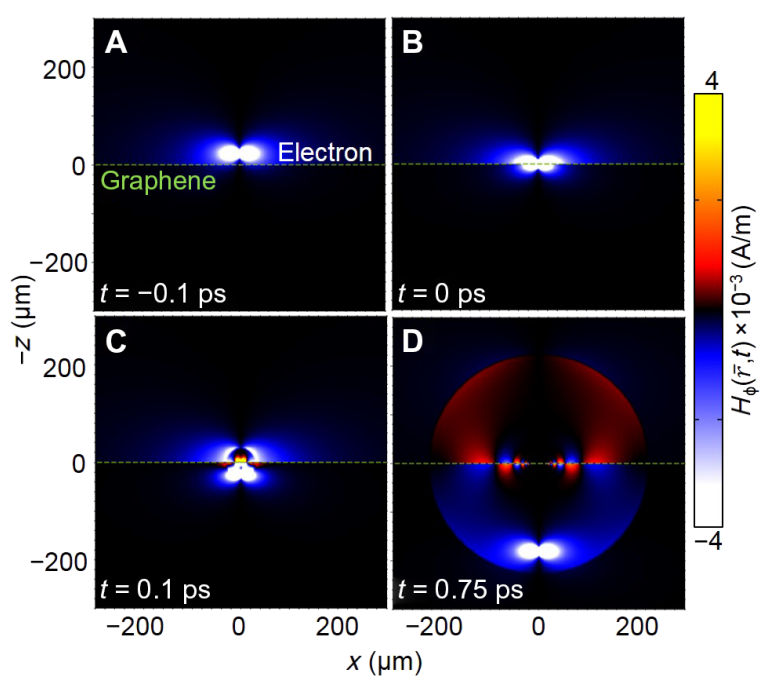

Fig. 2. Time evolution of magnetic field $H_{\phi}(\bar{r}, t)$ when a swift electron perpendicularly penetrates through a graphene monolayer. The green dashed line represents graphene. The electron is located (A) above graphene, (B) at graphene, and (C) below graphene.
As the water rushes back to fill the crater, a central jet column called the Rayleigh jet due to its instability (17), or the Worthington jet because Worthington performed extensive observations back in $1908(17,18)$, will rise above the initial water surface along the collapse axis. When it comes to charge motion on graphene, a "crater" of the density of conduction electron in graphene is first formed when the incident electron approaches the graphene layer (Fig. 3, A to C). Immediately after the penetration of the electron through the graphene layer, a rebound of the density of conduction electrons is formed with a central jet-like rise (Fig. 3, D and E), analogous to the hallmark of Rayleigh jet or Worthington jet in hydrodynamics. We observe, as in Fig. 3E, that the jet-like rise reaches its peak at about $t=0.15 \mathrm{ps}$. Other analogs of this hydrodynamic splashing jet have also been widely discussed, including those occurring during the meteor impact $(17,18)$ and during the interaction between the highly focused femtosecond laser pulses and a metal film surface (35).

Second, after the central jet-like rise falls down, ripples of $2 \mathrm{D}$ plasmons propagate outward as concentric circles, as shown in Fig. 3, F to $\mathrm{H}$. The excited 2D plasmons, which cover a broad spectrum of frequencies, gradually spread into a sequence of plasmonic ripples where longerwavelength plasmons stay at the outer periphery and shorter-wavelength plasmons stay closer to the inner boundary (Fig. $3 \mathrm{H}$ ). This is because the dispersion of $2 \mathrm{D}$ plasmons (including graphene plasmons) is typically $\omega \propto \sqrt{\kappa_{\perp}}(24,25)$, which is formally analogous to the dispersion of deep-water waves; this is reflected in the fact that longer wavelengths go out faster (see section S4), although they are formed later (for their longer formation times) than the shorter wavelengths.

\section{Energy of generated photons and 2D plasmons}

We plot in Fig. 4A the time evolution of total radiated photon energy by numerically monitoring the Poynting power going through an imaginary sphere with a large radius $R$ centered at the origin and then shifting the time axis backward by $R / c$, where the photon radiation is analogous to the sound emitted from a hydrodynamic splash. The total radiated photon energy eventually saturates and approaches a value of $0.171 \times$ $10^{-4} \mathrm{eV}$ that we calculate analytically by letting $t=\infty$ (see the Supplementary Materials). It can be seen that most photon energy has been radiated out before $t=0.15 \mathrm{ps}$, which is the moment when the jet-like rise of charge reaches its maximum (Fig. 3E) because most photon energy is shaken off by the charge jet. We also plot the time evolution of "energy" of the induced fields without considering the interference

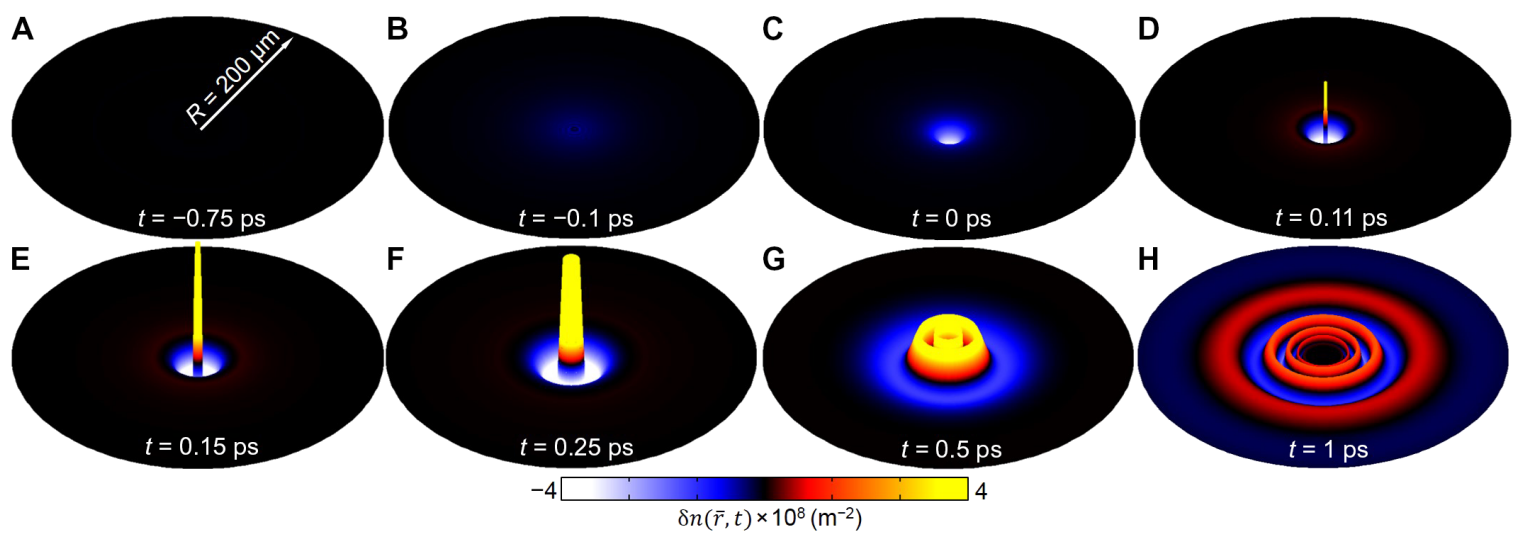

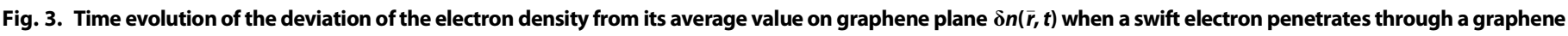
monolayer. The electron is located (A and B) above graphene, (C) at graphene, and (D to H) below graphene. 


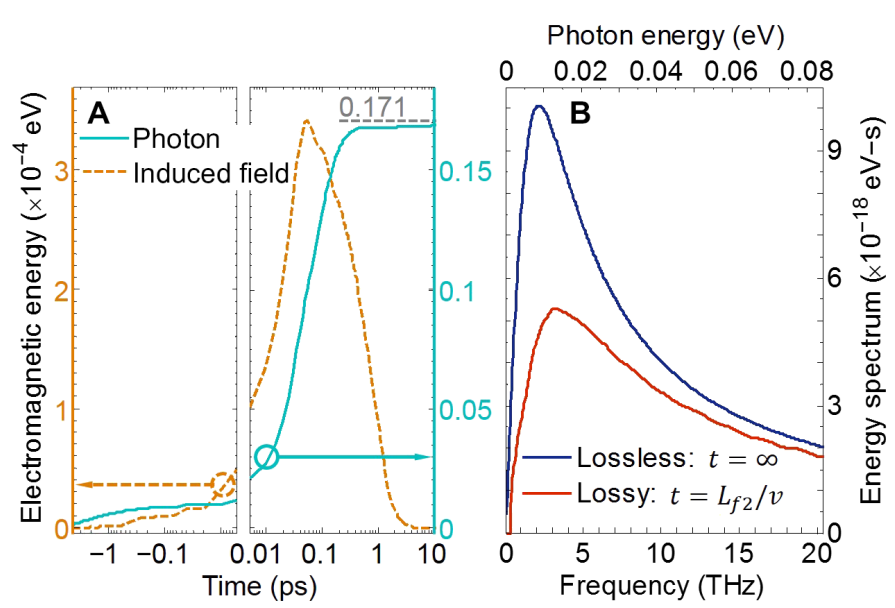

Fig. 4. Energy dissipation during the plasmonic formation time. (A) Time evolution of emitted photon energy and the induced field energy [related to the induced field strength $\left.\left(E_{\overline{\mathrm{K}}_{\perp}, \omega}^{1,2}\right)^{2}\right]$. (B) Energy spectra of graphene plasmons by taking $t=\infty$ in the lossless case and by taking $t=L_{f_{2}} / v$ in the lossy case.

fields of the swift electron. Note that the 2D plasmons have not been fully generated before their formation times, so this energy should be treated as a parameter related to the field strength $\left(E_{\overline{\mathrm{K}}_{\perp}, \omega}^{1,2}\right)^{2}$ of induced charges but not as the real energy. It can be seen that even before most photon energy is radiated out, the field strength of $\left(E_{\overline{\mathrm{\kappa}}_{\perp}, \omega}^{1,2}\right)^{2}$ has already started to drop.

We calculate the real energy of generated graphene plasmons by checking the electromagnetic energy for each frequency component immediately after the time $t=L_{f 2} / v$. For the calculation spectrum of 2D plasmons from 0 to $20 \mathrm{THz}$, the corresponding formation time $\left(L_{f 1}+\right.$ $\left.L_{f 2}\right) / v$ varies from $\infty$ to $0.1 \mathrm{ps}$, whose duration is comparable to the propagation time ( $\sim 0.5 \mathrm{ps})$ for graphene plasmons (see section S4). The energy loss during the formation time cannot be taken into account by simply resorting to the propagation time or propagation length of $2 \mathrm{D}$ plasmons that are widely used to characterize the propagation of graphene plasmons because this energy loss happens before the generation of 2D plasmons themselves.

Only in the ideal lossless situation can one equate the EEL spectrum to the energy spectrum of generated $2 \mathrm{D}$ plasmons (the energy of emitted photons generally occupies only $<5 \%$ of the energy loss of the swift electron, and considerably less for lower electron velocities, and thus is negligible). We plot in Fig. 4B the spectrum of 2D plasmons in the ideal lossless situation by taking $t=\infty$, which exactly agrees with the EEL spectrum calculation (see sections S5 and S6 and fig. S4). We also plot in Fig. 4B the spectrum of 2D plasmons for the realistic lossy graphene (the relaxation time is finite) after formation time is taken into account by taking $t=L_{f 2} / v$. The comparison between the lossless and lossy cases shows that a significant portion of energy (more than $25 \%$ total energy from 0 to $20 \mathrm{THz}$ ) has already been dissipated before $2 \mathrm{D}$ plasmons are generated. In addition, the losses in graphene, including those caused by defects, can be theoretically characterized by the relaxation time, where a smaller relaxation time indicates a larger loss. If more defects exist in the graphene sample, this will unavoidably degrade the relaxation time used in this work $(0.5 \mathrm{ps})$, resulting in a larger loss in graphene, and it is thus expected that a larger portion of energy will be dissipated before $2 \mathrm{D}$ plasmons are generated.

\section{DISCUSSION}

Recent advances in electron microscopy now suggest a new way to observe the temporal dynamics of the plasmons, including its ultrafast and subwavelength features, by using photon-induced near-field electron microscopy $(7,36)$. This exciting possibility will allow one to visualize the plasmonic splashing-like effects, instead of inferring them indirectly through EELS or transition radiation measurements.

Because graphene is just one typical example of many different $2 \mathrm{D}$ electron gas systems $(19-23,37,38)$ that support 2D plasmons, our analysis could be applicable to any 2D electron system. Therefore, other 2D electron gas systems (19-23), and particularly other 2D materials $(37,38)$, will likely further enrich the potential implementations of plasmonic splashing-like transients. However, we argue that graphene is particularly suitable for this purpose because of the flexible tunability of the chemical potential and the recent advances in the fabrication of graphene samples with large area, high quality, and the precise control of the layer number. In addition, it is reported that van der Waals materials can support different kinds of polaritons (38), such as phonon polaritons in a boron nitride slab $(37,38)$. We thus expect more polaritonic splashing-like transient to be revealed in van der Waals materials.

The above results demonstrate the transients of $2 \mathrm{D}$ plasmon generation in EELS experiment. An intermediate process between the impact of the swift electron and the formation of 2D plasmons is revealed as a result of the space-time limitation on plasmon generation. This process is found to be analogous to the hydrodynamic splashing phenomenon. Explicit calculation that takes into account the energy dissipation before $2 \mathrm{D}$ plasmons are generated has shown that previous estimates on the yields of graphene plasmons in EELS under the assumption of negligible loss need to be reevaluated.

\section{MATERIALS AND METHODS}

Our rigorous analytical calculations are based on an extension of Ginzburg and Frank's theory on transition radiation within the framework of macroscopic electrodynamics. This can give us an analytical expression of the radiated fields induced by swift electrons. From this, we can further analytically derive the spectrum of emitted photons; with the assumption of trivial losses in the calculated system, we can also analytically derive the EEL spectrum. Because the EEL spectrum is derived without the assumption of the electrostatic approximation, our analytical formula of the EEL spectrum is applicable to the cases with high electron kinetic energies. Furthermore, by choosing the appropriate Sommerfeld branch cut and using the Sommerfeld integration path, numerical calculation of the radiated fields at different times can be carried out. Therefore, the emitted photon energy, the induced field energy, and the energy spectrum of induced charges on lossy graphene at different times can be numerically obtained by applying these numerically calculated radiated fields.

\section{SUPPLEMENTARY MATERIALS}

Supplementary material for this article is available at http://advances.sciencemag.org/cgi/ content/full/3/1/e1601192/DC1

section S1. Figure caption of movie S1.

section S2. Photon emission from graphene affected by swift electrons.

section S3. Energy spectrum of induced charges on graphene.

section S4. Dispersion relation and propagation length/time of graphene plasmons. section S5. Analytical EEL spectrum.

section S6. Comparison of EEL spectra between previous work and our result. section S7. Total energy of emitted photons. 
section S8. Numerical implementation with Sommerfeld integration.

fig. S1. Dispersion curve of TM graphene plasmons.

fig. S2. Propagation time of TM graphene plasmons as a function of frequency.

fig. S3. EEL spectrum when an electron normally incident on an ideal lossless graphene layer.

fig. S4. EEL spectra for an electron normally incident on graphene.

movie S1. Time evolution of 2D plasmons launched by swift electrons.

References (39-52)

\section{REFERENCES AND NOTES}

1. F. J. Garcia de Abajo, Optical excitations in electron microscopy. Rev. Mod. Phys. 82, 209-275 (2010).

2. R. H. Ritchie, Plasma losses by fast electrons in thin films. Phys. Rev. 106, 874 (1957).

3. F. J. Garcia de Abajo, Multiple excitation of confined graphene plasmons by single free electrons. ACS Nano 7, 11409-11419 (2013).

4. Y. Liu, R. F. Willis, K. V. Emtsev, T. Seyller, Plasmon dispersion and damping in electrically isolated two-dimensional charge sheets. Phys. Rev. B 78, 201403 (2008).

5. R. J. Koch, T. Seyller, J. A. Schaefer, Strong phonon-plasmon coupled modes in the graphene/silicon carbide heterosystem. Phys. Rev. B 82, 201413 (2010).

6. S. Y. Shin, N. D. Kim, J. G. Kim, K. S. Kim, D. Y. Noh, K. S. Kim, J. W. Chung, Control of the $\pi$ plasmon in a single layer graphene by charge doping. Appl. Phys. Lett. 99, 082110 (2011).

7. L. Piazza, T. T. A. Lummen, E. Quiñonez, Y. Murooka, B. W. Reed, B. Barwick, F. Carbone, Simultaneous observation of the quantization and the interference pattern of a plasmonic near-field. Nat. Commun. 6, 6407 (2015).

8. W. Zhou, J. Lee, J. Nanda, S. T. Pantelides, S. J. Pennycook, J. C. Idrobo, Atomically localized plasmon enhancement in monolayer graphene. Nat. Nanotechnol. 7, 161-165 (2012)

9. J. Song, L. Zhang, Y. Xue, Q. Y. S. Wu, F. Xia, C. Zhang, Y.-L. Zhong, Y. Zhang, J. Teng, M. Premaratne, C.-W. Qiu, Q. Bao, Efficient excitation of multiple plasmonic modes on three-dimensional graphene: An unexplored dimension. ACS Photon. 3, 1986-1992 (2016).

10. Y. Lu, J. Song, J. Yuan, L. Zhang, S. Q. Y. Wu, W. Yu, M. Zhao, C.-W. Qiu, J. Teng, K. P. Loh, C. Zhang, Q. Bao, Highly efficient plasmon excitation in graphene-Bi2Te3 heterostructure. J. Opt. Soc. Am. B 33, 1842-1846 (2016).

11. M. Zhao, M. Bosman, M. Danesh, M. Zeng, P. Song, Y. Darma, A. Rusydi, H. Lin, C.-W. Qiu, K. P. Loh, Visible surface plasmon modes in single $\mathrm{Bi}_{2} \mathrm{Te}_{3}$ nanoplate. Nano Lett. 15, 8331-8335 (2015).

12. V. L. Ginzburg, Transition radiation and transition scattering. Phys. Scripta T2, 182-191 (1982).

13. V. L. Ginzburg,V. N. Tsytovich, Transition Radiation and Transition Scattering (CRC Press, 1990).

14. U. I. Uggerhøj, The interaction of relativistic particles with strong crystalline field. Rev. Mod. Phys. 77, 1131-1171 (2005).

15. I. M. Khalatnikov, J. B. Sykes, Landau, the Physicist and the Man: Recollections of L.D. Landau (Pergamon Press, 1989).

16. N. Bohr, The quantum postulate and the recent development of atomic theory. Nature 121, 580-590 (1928).

17. M. Rein, Phenomena of liquid drop impact on solid and liquid surface. Fluid Dyn. Res. 12, 61-93 (1993).

18. A. M. Worthington, A Study of Splashes (Longmans, Green and Co, 1908).

19. F. Stern, Polarizability of a two-dimensional electron gas. Phys. Rev. Lett. 18, 546 (1967).

20. A. Eguiluz, T. K. Lee, J. J. Quinn, K. W. Chiu, Interface excitations in metal-insulatorsemiconductor structures. Phys. Rev. B 11, 4989 (1975).

21. A. V. Chaplik, Possible crystallization of charge carriers in low-density inversion layers. J. Exp. Theor. Phys. 62, 746-753 (1972).

22. T. N. Theis, J. P. Kotthaus, P. J. Stiles, Wavevector dependence of the two-dimensional plasmon dispersion relationship in the (100) silicon inversion layer. Solid State Commun. 26, 603-606 (1978).

23. S. D. Sarma, A. Madhukar, Collective modes of spatially separated, two-component, two-dimensional plasma in solids. Phys. Rev. B 23, 805-815 (1981).

24. A. N. Grigorenko, M. Polini, K. S. Novoselov, Graphene plasmonics. Nat. Photonics 6 , 749-758 (2012).

25. M. Jablan, H. Buljan, M. Soljačić, Plasmonics in graphene at infrared frequencies. Phys. Rev. $B$ 80, 245435 (2009).

26. M. Dyakonov, M. Shur, Shallow water analogy for a ballistic field effect transistor: New mechanism of plasma wave generation by dc current. Phys. Rev. Lett. 71, 2465 (1993).
27. D. Svintsov, V. Vyurkov, V. Ryzhii, T. Otsuji, Hydrodynamic electron transport and nonlinear waves in graphene. Phys. Rev. B 88, 245444 (2013).

28. A. O. Govorov, J. J. Heremans, Hydrodynamic effects in interacting Fermi electron jets. Phys. Rev. Lett. 92, 026803 (2004).

29. A. P. Dmitriev, A. S. Furman, V. Yu. Kachorovskii, G. G. Samsonidze, Numerical study of the current instability in a two-dimensional electron fluid. Phys. Rev. B 55, 10319 (1997).

30. V. P. Gusynin, S. G. Sharapov, J. P. Carbotte, Unusual microwave response of Dirac quasiparticles in graphene. Phys. Rev. Lett. 96, 256802 (2006).

31. L. A. Falkovsky, A. A. Varlamov, Space-time dispersion of graphene conductivity. Eur. Phys. J. B. 56, 281-284 (2007).

32. U. N. Maiti, W. J. Lee, J. M. Lee, Y. Oh, J. Y. Kim, J. E. Kim, J. Shim, T. H. Han, S. O. Kim, 25th anniversary article: Chemically modified/doped carbon nanotubes \& graphene for optimized nanostructures \& nanodevices. Adv. Mater. 26, 40-67 (2014).

33. A. Woessner, M. B. Lundeberg, Y. Gao, A. Principi, P. Alonso-González, M. Carrega, K. Watanabe, T. Taniguchi, G. Vignale, M. Polini, J. Hone, R. Hillenbrand, F. H. L. Koppens, Highly confined low-loss plasmons in graphene-boron nitride heterostructures. Nat. Mater. 14, 421-425 (2015).

34. N. Yamamoto, K. Araya, A. Toda, H. Sugiyama, Light emission from surfaces, thin films and particles induced by high-energy electron beam. Surf. Interface Anal. 31, 79-86 (2001).

35. V. K. Valev, D. Denkova, X. Zheng, A. I. Kuznetsov, C. Reinhardt, B. N. Chichkov, G. Tsutsumanova, E. J. Osley, V. Petkov, B. D. Clercq, A. V. Silhanek, Y. Jeyaram, V. Volskiy, P. A. Warburton, G. A. E. Vandenbosch, S. Russev, O. A. Aktsipetrov, M. Ameloot, V. V. Moshchalkov, T. Verbiest, Plasmon-enhanced sub-wavelength laser ablation: plasmonic nanojets. Adv. Mater. 24, OP29-OP35 (2012).

36. B. Barwick, D. J. Flannigan, A. H. Zewail, Photon-induced near-field electron microscopy. Nature 462, 902-906 (2009).

37. F. Xia, H. Wang, D. Xiao, M. Dubey, A. Ramasubramaniam, Two-dimensional material nanophotonics. Nat. Photon. 8, 899-907 (2014).

38. D. N. Basov, M. M. Fogler, F. J. García de Abajo, Polaritons in van der Waals materials. Science 354, aag1992 (1992).

39. V. P. Gusynin, S. G. Sharapov, J. P. Carbotte, Magneto-optical conductivity in graphene. J. Phys. Condens. Mat. 19, 026222 (2007).

40. S. A. Mikhailov, K. Ziegler, New electromagnetic mode in graphene. Phys. Rev. Lett. 99, 016803 (2007).

41. G. W. Hanson, Dyadic Green's functions and guided surface waves for a surface conductivity model of graphene. J. Appl. Phys. 103, 064302 (2008).

42. Z. Fei, A. S. Rodin, G. O. Andreev, W. Bao, A. S. McLeod, M. Wagner, L. M. Zhang, Z. Zhao, M. Thiemens, G. Dominguez, M. M. Fogler, A. H. Castro Neto, C. N. Lau, F. Keilmann, D. N. Basov, Gate-tuning of graphene plasmons revealed by infrared nano-imaging Nature 487, 82-85 (2012).

43. V. L. Ginzburg, V. N. Tsytovich, Several problems of the theory of transition radiation and transition scattering. Phys. Rep. 49, 1-89 (1979).

44. J. D. Jackson, Classical Electrodynamics (John Wiley \& Sons, 1999).

45. L. D. Landau, E. M. Lifshitz, Electrodynamics of Continuous Media (Pergamon Press, 1984).

46. E. H. Hwang, S. Das Sarma, Dielectric function, screening, and plasmons in two-dimensional graphene. Phys. Rev. B 75, 205418 (2007).

47. G. B. Whitham, Linear and Nonlinear Waves (John Wiley \& Sons, 1974).

48. W. C. Chew, Waves and Fields in Inhomogeneous Media (IEEE Press, 1995).

49. X. Lin, R. Li, F. Gao, E. Li, X. Zhang, B. Zhang, H. Chen, Loss induced amplification of graphene plasmons. Opt. Lett. 41, 681-684 (2016).

50. J. V. Jelly, Cerenkov Radiation and Its Applications (Pergamon Press, 1958).

51. B. Zhang, B.-I. Wu, Electromagnetic detection of a perfect invisibility cloak. Phys. Rev. Lett. 103, 243901 (2009).

52. H. Chen, M. Chen, Flipping photons backward: Reversed Cherenkov radiation. Mater. Today 14, 34-41 (2011).

Acknowledgments: We thank L. Lu for helpful discussions. Funding: This work was sponsored by the National Natural Science Foundation of China (grant nos. 61625502, 61574127, 61601408, and 61550110245), the Zhejiang Natural Science Foundation (grant no. LY17F010008), the Postdoctoral Science Foundation of China (grant no. 2015M581930), the Top-Notch Young Talents Program of China, the Innovation Joint Research Center for Cyber-Physical-Society System, the Nanyang Technological University for Nanyang Assistant Professorship Start-Up Grant, the Singapore Ministry of Education (grant nos. MOE2015-T2-1-070 and MOE2011-T3-1-005), the U.S. Army Research Laboratory and the U.S. Army Research Office through the Institute for Soldier Nanotechnologies (contract no. W911NF-13-D-0001), the Massachusetts Institute of Technology S3TEC Energy Frontier Research Center of the Department of Energy (grant no. de-sc0001299), the QuantiXLie Center of Excellence, and the Chinese Scholarship Council (CSC 201506320075). Author contributions: All authors contributed extensively to this work. B.Z. conceived the idea. 
X.L. performed the main calculations. X.S., Z.Y., F.G., and Z.G. contributed to the numerical Sommerfeld integration and the analytical calculations on photon radiation. I.K. contributed to the EEL spectrum calculation. X.L., I.K., H.B., J.D.J., M.S., H.C., and B.Z. analyzed the data and discussed and interpreted the detailed results. I.K., H.C., and B.Z. supervised the project. All authors discussed, revised, and approved the manuscript. Competing interests: The authors declare that they have no competing interests. Data and materials availability: All data needed to evaluate the conclusions in the paper are present in the paper and/or the Supplementary Materials. Additional data related to this paper may be requested from the authors.
Submitted 26 May 2016

Accepted 19 December 2016

Published 27 January 2017

$10.1126 /$ sciadv. 1601192

Citation: X. Lin, I. Kaminer, X. Shi, F. Gao, Z. Yang, Z. Gao, H. Buljan, J. D. Joannopoulos, M. Soljačić, H. Chen, B. Zhang, Splashing transients of 2D plasmons launched by swift electrons. Sci. Adv. 3, e1601192 (2017). 


\section{ScienceAdvances}

\section{Splashing transients of 2D plasmons launched by swift electrons}

Xiao Lin, Ido Kaminer, Xihang Shi, Fei Gao, Zhaoju Yang, Zhen Gao, Hrvoje Buljan, John D. Joannopoulos, Marin Soljacic, Hongsheng Chen and Baile Zhang

Sci Adv 3 (1), e1601192

DOI: $10.1126 /$ sciadv.1601192

ARTICLE TOOLS

SUPPLEMENTARY MATERIALS

REFERENCES

PERMISSIONS http://advances.sciencemag.org/content/3/1/e1601192

http://advances.sciencemag.org/content/suppl/2017/01/23/3.1.e1601192.DC1

This article cites 44 articles, 0 of which you can access for free http://advances.sciencemag.org/content/3/1/e1601192\#BIBL

http://www.sciencemag.org/help/reprints-and-permissions

Use of this article is subject to the Terms of Service 